OPEN ACCESS

Edited by:

Xiang Cheng,

Huazhong University of Science and Technology, China

Reviewed by:

Shrivani Pirahas,

University of Calgary, Canada

Daiju Fukuda,

Tokushima University, Japan

*Correspondence:

Xian Wu Cheng

chengxw0908@163.com

Xiang Li

zhongtian200330@163.com

${ }^{\dagger}$ These authors have contributed equally to this work

Specialty section:

This article was submitted to

Signaling,

a section of the journal

Frontiers in Cell and Developmental

Biology

Received: 30 March 2021

Accepted: 04 June 2021

Published: 21 July 2021

Citation:

Lei Y, Cui Q, Yang G, Piao L, Inoue $A, W u H, L i X$, Kuzuya $M$ and

Cheng XW (2021) Statins Mitigate

Stress-Related Vascular Aging and Atherosclerosis in apoE-Deficient

Mice Fed High Fat-Diet: The Role of Glucagon-Like

Peptide-1/Adiponectin Axis. Front. Cell Dev. Biol. 9:687868. doi: 10.3389/fcell.2021.687868

\section{Statins Mitigate Stress-Related Vascular Aging and Atherosclerosis in apoE-Deficient Mice Fed High Fat-Diet: The Role of Glucagon-Like Peptide-1/Adiponectin Axis}

\author{
Yanna Lei ${ }^{1+}$, Qingsong Cui ${ }^{1+}$, Guang Yang ${ }^{2}$, Limei Piao ${ }^{2}$, Aiko Inoue ${ }^{3}$, Hongxian $W^{4}$, \\ Xiang $\mathrm{Li}^{1 *}$, Masafumi Kuzuya ${ }^{3,5}$ and Xian Wu Cheng ${ }^{1,3 *}$ \\ ${ }^{1}$ Department of Intensive Care Unit, Yanbian University Hospital, Yanjin, China, ${ }^{2}$ Department of Cardiology \\ and Hypertension, Yanbian University Hospital, Yanjin, China, ${ }^{3}$ Institute of Innovation for Future Society, Nagoya University \\ Graduate School of Medicine, Nagoya, Japan, ${ }^{4}$ Department of Cardiology, Shanghai Institute of Cardiovascular Disease, \\ Zhongshan Hospital, Fudan University, Shanghai, China, ${ }^{5}$ Department of Community Healthcare \& Geriatrics, Nagoya \\ University Graduate School of Medicine, Nagoya, Japan
}

Objectives: Exposure to chronic psychosocial stress is a risk factor for atherosclerotic cardiovascular diseases. Given that the 3-hydroxy-3-methylglutarylcoenzyme reductase inhibitor statins prevent atherogenesis, we evaluated whether pitavastatin prevents chronic stress- and high fat diet-induced vascular senescence and atherogenesis in apolipoprotein $E$-deficient $\left(A p o E^{-/-}\right)$mice, with a special focus on glucagon-like peptide-1 (GLP-1)/adiponectin (APN) axis.

Methods and Results: 6-week-old $A p o E^{-/-}$mice loaded a high-fat diet were randomly assigned into non-stress $(n=12)$ and stress $(n=13)$ groups for 12 weeks. Non-stress control mice were left undisturbed. Chronic stress accelerated high fat diet-induce arterial senescence and atherosclerotic plaque growth. The chronic stress lowered the levels of circulating GLP-1 as well as adipose and plasma APN. As compared with the stress alone mice, the pitavastatin-treated mice had reduced macrophage infiltration, elastin fragments, and increased plaque collagen volume, and lowered levels of osteopontin, toll-like receptor-2/-4, macrophage chemoattractant protein-1, C-X-C chemokine receptor-4, p47 ${ }^{\text {phox }}$, p47 ${ }^{\text {phox }}$, gp91 ${ }^{\text {phox }}$, cathepsins S, p16, and p21, mRNAs and/or proteins. Pitavastatin increased plasma GLP-1 and APN levels and suppressed matrix metalloproteinase-2/-9 gene expressions and activities in the aortas. Finally, the protective effect of pitavastatin was abrogated by APN blocking.

Conclusion: These findings suggested that the pitavastatin-mediated pleiotropic vasculoprotective effects are likely attributable, at least in part, to the elevation of GLP-1 and APN levels and the inhibition of diet-induced plaque inflammation, oxidative stress, and proteolysis in $A p o E^{-/-}$mice received chronic stress conditions.

Keywords: chronic stress, statins, atherosclerosis, inflammation, adiponectin 


\section{INTRODUCTION}

Accumulating evidence indicated that chronic stress is involved in metabolic and inflammatory cardiovascular disorders (Bernberg et al., 2012; Heidt et al., 2014). The mechanisms underlying chronic stress-related diseases have thus become a research focus. At present, most of the evidence suggests that the pathogenic effect of chronic stress is exerted mainly on the hypothalamic-pituitary-adrenal axis and/or the body's sympathetic nervous system, followed by the induction of disorders such as atherosclerosis (Barik et al., 2013; Cox et al., 2014; Heidt et al., 2014). Recently we demonstrated that chronic stress can prompt the formation of atherosclerosis by accelerating inflammation and oxidative stress (Lei et al., 2017; Yang et al., 2017). Despite the large number of studies that have been performed, the precise mechanisms are not yet clear, and effective strategies to cope with chronic stress-related diseases remain to be established.

Statins are traditional anti-atherosclerosis drugs, although they are normally used against low-density lipoprotein cholesterol (LDL-C); statins have also been shown to have an anti-atherosclerotic effect partly by inhibiting inflammation and oxidative stress (Lin et al., 2015). Statins have been widely adopted as a clinical atherosclerosis treatment, but the question of whether statins also have a beneficial effect on chronic stressrelated atherosclerosis has not been resolved. We conducted the present study to explore the potential mechanisms involved in chronic stress-related atherosclerosis formation, focusing on inflammation and oxidative stress. We also investigated whether the traditional anti-atherosclerosis drug pitavastatin can ameliorate this stress-related atherosclerosis and its mechanisms, with a special focus on glucagon-like peptide-1 (GLP-1)/adiponectin (APN) axis.

\section{MATERIALS AND METHODS}

\section{Animals}

Four-week-old male $A p o E^{-/-}$mice (21-24 g body weight; KOR/StmSlc-Apoe ${ }^{\text {shl }}, \mathrm{BALB} / \mathrm{c}$ background) were purchased from Chubu Science and Material Science Company (SLC, Hamamatsu, Japan). For their adaptation to the new environment, the mice were all fed a standard diet for 2 weeks and housed two per cage under standard conditions (50 $\pm 5 \%$ humidity, $23 \pm 1^{\circ} \mathrm{C}$ ). The animal experimental protocols were approved by the Institutional Animal Care and Use Committee of Nagoya University (Protocol No. 27304) and of Yanbian University (Protocol No. 2018-10) and performed according to the Guide for the Care and Use of Laboratory Animals published by the U.S. National Institutes of Health.

\section{Mouse Immobilized Stress Protocol and Tissue Correction}

The 6-week-old male $A p o E^{-/-}$mice $(n=25)$ were randomly assigned into the following two groups for the first phase of the experiment: The control group (HF-C, $n=12$ ) received a highfat (HF) diet ( $21.00 \%$ fat and $0.15 \%$ cholesterol) only, and the stress group (HF-S, $n=13$ ) received the same HF diet and were subjected to restraint stress (12 weeks) (Lei et al., 2017). For the second phase of the experiment, the $A p o E^{-/-}$mice fed a HF diet were divided to another two groups: Stress 2 group (Stress): the mice subjected to restraint stress for 12 weeks as mentioned above, and the stress + pitavastatin group (S-Pis): Except for HF diet and restraint stress (12 weeks), the mice were treated with pitavastatin $(1 \mathrm{mg} / \mathrm{kg} / \mathrm{d})$. The pitavastatin used in this experiment was provided by Kowa Pharmaceutical Co. Ltd. (Nagoya, Japan). For the APN deletion examinations, the mice $(n=10)$ fed with HF-diet were divided to one of two groups and gave pitavastatin + control rabbit IgG (Control, $450 \mu \mathrm{g} / \mathrm{kg} / \mathrm{d}$, ab27472, Cambridge, United Kingdom) or pitavastatin plus neutralizing rabbit APN antibody (S-NAPN, $450 \mu \mathrm{g} / \mathrm{kg} / \mathrm{d}$, ab3455) given by subcutaneous injection every week under chronic stress conditions for 8 weeks.

After euthanasia of the $A p o E^{-/-}$mice by means of an overdose of pentobarbital (50 mg/kg; Dainippon Pharmaceutical, Osaka, Japan) at the end of stress/no stress treatment, blood samples were collected just before perfusion into syringes containing heparin. After perfusion with $0.01 \mathrm{M}$ phosphatebuffered saline (PBS, pH 7.4), the aorta tissues were collected for biological analyses and histological characterization analyses.

\section{Histological Analysis}

The cross-sections of the aortas were examined as we described (Cheng et al., 2011). The heart was sliced in a plane between the lower tips of the left and right atria, and the upper portion of the heart was isolated. The hearts were kept in $4 \%$ formalin. Serial sections (3- $\mu \mathrm{m}$ thick at $15-\mu \mathrm{m}$ intervals) were isolated on slides for morphological analysis and immunostaining.

\section{Quantitative Real-Time PCR}

Total RNA was extracted from the aortic tissues and preadipocytes with the use of the RNeasy Fibrous Tissue MiNi-Kit (Qiagen, Hilden, Germany) and was subjected to reverse transcription following the manufacturer's instructions. The cDNA was generated by the SuperScript III CellsDirect cDNA Synthesis kit (Invitrogen, Carlsbad, CA, United States). A polymerase chain reaction (PCR) was done by the ABI 7300 Real-time PCR System (Applied Biosystems, Foster City, CA, United States). The expression of glyceraldehyde 3-phosphate dehydrogenase (GAPDH) was used as an internal standard for each targeted gene levels. The primer sequences for the macrophage chemoattractant protein-1 (MCP-1), toll-like receptor-2/-4 (TLR-2/-4), p47 ${ }^{\text {phox }}$, p47 $^{\text {phox }}$, gp91 ${ }^{\text {phox }}$, cathepsins $S$ (Cat S), matrix metalloproteinase-2/-9, (MMP-2/-9), and C-X-C chemokine receptor-4 (CXCR-4) are listed in Table 1.

\section{Immunohistochemistry}

The 3- $\mu \mathrm{m}$-thick paraffin sections of aortas were H\&E stained as we described (Cheng et al., 2011). Corresponding slides were immunostained with mouse antibodies against macrophages (CD68; 1:100, Chemicon, Billerica, MA, United States), mouse antibodies against osteopontin (1:125, Sigma-Aldrich, St. Louis, $\mathrm{MO}$, United States), rabbit antibodies against alpha-smooth muscle actin ( $\alpha$-SMA; 1:100, Neo Markers, Fremont, CA, United States). Elastin and collagen contents were quantified using the Elastica van Gieson (EVG)-stained and picrosirius 
TABLE 1 | Primer sequences for mice used for quantitative real-time PCR.

\begin{tabular}{|c|c|c|}
\hline $\begin{array}{l}\text { Genes } \\
\text { name }\end{array}$ & Forward primers & Reverse primers \\
\hline MMP-2 & CCCCATGAAGCCTTGTTTACC & TTGTAGGAGGTGCC CTGGAA \\
\hline MMP-9 & $\begin{array}{l}\text { CCAGACGCTCTTCGA } \\
\text { GAACC }\end{array}$ & GTTATAGAAGTGGC GGTTGT \\
\hline Cat S & GTGGCCACTAAAGG GCCTG & $\begin{array}{l}\text { ACCGCTाTGTAGAAGAAGA } \\
\text { AGGAG }\end{array}$ \\
\hline gp 91 & $\begin{array}{l}\text { ACTTCCATAAGATGGTAGC } \\
\text { TTGG }\end{array}$ & $\begin{array}{l}\text { GCATTCACACACCAC } \\
\text { TCAACG }\end{array}$ \\
\hline MCP-1 & $\begin{array}{l}\text { GCCCCACTCACCTGC } \\
\text { TGCTACT }\end{array}$ & $\begin{array}{l}\text { CCTGCTGCTGGTGATCC } \\
\text { TCTTGT }\end{array}$ \\
\hline TLR-2 & $\begin{array}{l}\text { AAGAAGCTGGCATTC } \\
\text { CGAGGC }\end{array}$ & $\begin{array}{l}\text { CGTCTGACTCCGAGGG } \\
\text { GTTGA }\end{array}$ \\
\hline TLR-4 & $\begin{array}{l}\text { AGTGGGTCAAGGAACA } \\
\text { GAAGCA }\end{array}$ & $\begin{array}{l}\text { CПTACCAGCTCATाT } \\
\text { СTCACC }\end{array}$ \\
\hline CXCR- 4 & $\begin{array}{l}\text { CCACCCAGGACAGTGTGACTCT } \\
\text { AA }\end{array}$ & $\begin{array}{l}\text { GATGGGATTTCTGTAT } \\
\text { GAGGATT }\end{array}$ \\
\hline p47 phox & $\begin{array}{l}\text { CTGAGGGTGAAGCCA } \\
\text { TTGAGG }\end{array}$ & GCCGGTGATATC CCC $\Pi T C C$ \\
\hline p67 phox & $\begin{array}{l}\text { AACTACCTGGAGCC } \\
\text { AGTTGAG }\end{array}$ & $\begin{array}{l}\text { AATTAGGAGGTGGTGGAAT } \\
\text { ATCGG }\end{array}$ \\
\hline
\end{tabular}

red (PSR)-stained positive areas. Images of sections stained for macrophages, elastin, osteopontin, $\alpha$-SMC, and collagen were quantified with ImagePro software (BZ9000 Analysis, Keyence, Japan). Six cross-sections of each aortas were calculated and averaged for each mouse. The results are expressed as the percentage of intima area that contained the lesion.

\section{Oil Red O Staining and Senescence-Associated $\beta$-Galactosidase ( $\beta$-Gal) Staining}

The whole aortas from mice of each group were selected. We first removed the extra fatty and adventitia tissue from the vessels using forceps and scissors. The vessels were then incised longitudinally along the artery and fixed in formalin overnight for oil red $\mathrm{O}$ staining, as described in our previous study (Lei et al., 2017). Images of each vessel were obtained, and the extent of atherosclerosis was determined with the ImagePro software. $\beta$-Gal staining and quantitative analyses were made as described with our previous study (Lei et al., 2017).

\section{ELISA and Biochemical Assays}

According to manufacturers' instructions, the plasma GLP-1 and adiponectin (APN) levels were evaluated using the commercially available ELISA kits (GLP-1, Cat. EZGLP1T-36K; EMD Millipore, Billerica, MA, United States; APN, Cat. MRP300; R\&D Systems, Minneapolis, MN, United States). The levels of the mouse plasma non-esterified fatty acid (NEFA), high-density lipoprotein cholesterol (HDL-C), low-density lipoprotein cholesterol (LDLC), glucose, triglyceride (TG), creatinine, and blood urea nitrogen (BUN) were evaluated at a commercial laboratory (SRL, Tokyo).

\section{Gelatin Zymography}

For gelatin zymography, $20 \mu \mathrm{g}$ of aortic protein extract was mixed with sodium dodecyl sulfate (SDS) sample buffer without reducing agent and loaded onto a 10\% SDS-polyacrylamide gel containing gelatin $(1 \mathrm{mg} / \mathrm{mL})$ containing $1 \mathrm{mg} / \mathrm{mL}$ gelatin as described as described in Lei et al. (2017). Following staining and destaining with the related buffers, the areas of gelatinolytic activity were visualized under a light microscope and the digestion bands were measured by an image analyzer software program (NIH Image 1.62).

\section{Western Blot Analysis}

The total aortic protein was extracted with lysis buffer. The DC protein assay kit (Bio-Rad Laboratories, Hercules, CA, United States) was applied to measure the concentration of each sample. The protein abundance was detected with antibodies against Sirt-1 (cat. no. 2028, Cell Signaling Technology, Danvers, MA, United States), p16 ${ }^{I N K 4 A}$ (CDKN2A, cat. no. 10883-1AP, Proteintech Group Inc., Rosemont, IL, United States), adiponectin receptor-1 (AdipR-1, ab70362), $\beta$-actin monoclonal antibody (1: 1000, AC-15, Sigma-Aldrich), and p21 (ab109199, both from Abcam, Cambridge, United Kingdom). The membranes then were incubated with the secondary antibodies. The protein contents calculated from western blots were normalized by loading $\beta$-actin.

\section{Preadipocyte Isolation and Culture}

Inguinal adipose tissues of the non-stress and stressed micederived immature adipocytes were prepared as described previously (Hao et al., 2014). The preadipocytes were cultured in Dulbecco's modified Eagle's medium (DMEM)/10\% fetal bovine serum (FBS), antibiotics (penicillin/streptomycin), and $4.5 \mathrm{~g} / \mathrm{l}$ glucose in a humidified atmosphere $\left(95 \%\right.$ air and $\left.5 \% \mathrm{CO}_{2}\right)$ at $37^{\circ} \mathrm{C}$. Following culturing in serum-free medium for $12 \mathrm{~h}$, the cells were applied to a biological assay.

\section{Statistical Analysis}

The data are expressed as the mean \pm standard error of the mean (SEM). We used Student's $t$-test for comparisons of two groups, and we conducted a one-way analysis of variance (ANOVA) for comparisons of three or more groups followed by Tukey's post hoc test. Probability $(p)$-values $<0.05$ were considered significant. All of the examinations were performed by two observers blinded to the treatment of the animals.

\section{RESULTS}

\section{Effects of Chronic Stress on Body Weight, Plasma Lipid Profile, and Plasma GLP-1/APN Axis}

The mice were weighed weekly and as expected, chronic stress significantly reduced the body weights of the stressed mice in a time-dependent manner compared to the non-stressed mice (Figure 1A) and also reduced the weight of subcutaneous and inguinal fat (Figures 1C,D). As shown in Table 2, except for 


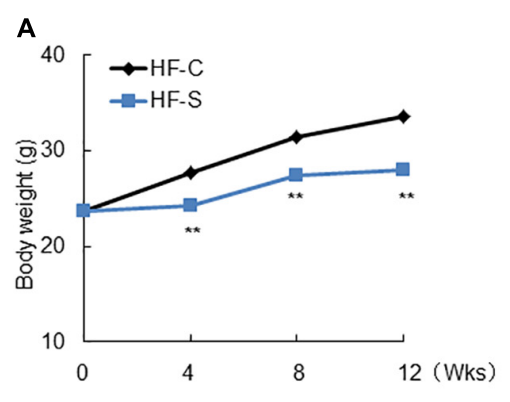

C

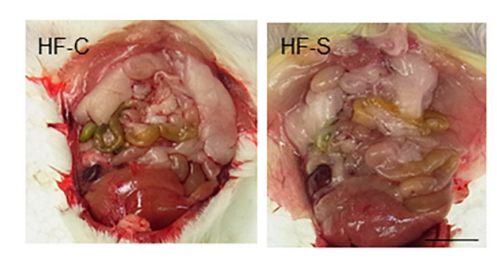

B

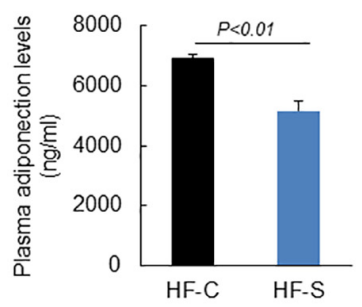

D

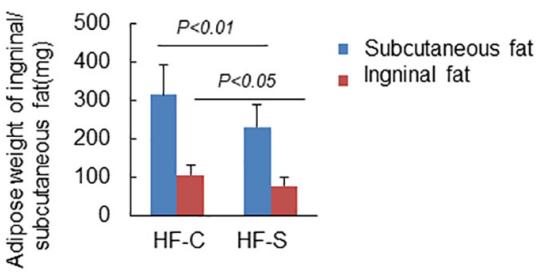

FIGURE 1 | Body weight (BW), adipose weight and adiponectin levels in the two experimental groups during the experimental period. (A) Chronic stress reduced BW in a time dependent manner. (B) Chronic stress decreased serum APN levels. (C,D) Representative images show the inguinal fat, and chronic stress reduced the weight of subcutaneous and inguinal fat. Data are presented as means \pm SEM $(n=6-8)$. $P<0.05$ was considered statistically significant by Student's un-paired t-test. Scale bars: $200 \mu \mathrm{m}$ (D).

TABLE 2 | Levels of lipids, GLP-1 activity, and other biochemical parameters.

\begin{tabular}{lcc}
\hline Parameter & HF-C & HF-S \\
\hline TG $(\mathrm{mg} / \mathrm{dL})$ & $188.0 \pm 4.7$ & $113.5 \pm 11.6^{\star}$ \\
LDL $(\mathrm{mg} / \mathrm{dL})$ & $224.0 \pm 18.4$ & $228.5 \pm 20.4$ \\
HDL $(\mathrm{mg} / \mathrm{dL})$ & $28.0 \pm 5.6$ & $26.5 \pm 4.8$ \\
NEFA $(\mu \mathrm{EQ} / \mathrm{L})$ & $882.5 \pm 26.3$ & $793.5 \pm 21.3$ \\
Glucose $(\mathrm{mg} / \mathrm{dL})$ & $172.0 \pm 16.4$ & $161.5 \pm 14.7$ \\
BUN $(\mathrm{mg} / \mathrm{dL})$ & $18.0 \pm 2.7$ & $18.0 \pm 3.1$ \\
CREA $(\mathrm{mg} / \mathrm{dL})$ & $0.5 \pm 0.0$ & $0.5 \pm 0.0$ \\
GLP-1 $(\mathrm{pM})$ & $15.2 \pm 0.9$ & $11.7 \pm 1.2^{\star \star}$ \\
\hline
\end{tabular}

TG, triglyceride; LDL, high-density lipoprotein; HDL, high-density lipoprotein; BUN, blood urine nitrogen; CREA, creatinine; NEFA, non-esterified fatty acid; GLP-1, glucagon-like peptide-1. All results are presented as means $\pm S E M,{ }^{*} P<0.05$, ${ }^{* *} P<0.01$ vs. corresponding controls by Student's un-paired t-test.

TG levels, chronic stress had no effect on other plasma lipid profile levels or the blood glucose and BUN, CREA levels. However, the plasma ANP and GLP-1 levels of the stressed mice were significantly decreased as compared to the non-stress mice (APN: $6904.4 \pm 124.3 \mathrm{ng} / \mathrm{ml}$ vs. $5167.1 \pm 301.7 \mathrm{ng} / \mathrm{ml}$; GLP-1: $15.2 \pm 0.9$ pM vs. $11.7 \pm 1.2 \mathrm{pM}$, respectively; $p<0.01$, Figure $1 \mathrm{~B}$ and Table 2).

\section{Chronic Stress Accelerated the Diet-Induced Lipid Accumulation, Inflammatory Response and Oxidative Stress of Atherosclerotic Lesions}

As we expected, the chronic stress significantly increased the atherosclerotic lesion area in the aortic root compared to the control values (atherosclerotic area: $521.3 \pm 57.6 \times 10^{3}{\mu \mathrm{m}^{2}}^{2}$ vs. $285.1 \pm 36.2 \% \times 10^{3} \mu \mathrm{m}^{2}, p<0.01$, Figures 2A,B), indicating that the chronic stress promoted atherosclerotic plaque expansion. Lipid deposition is known to be an initial process of atherosclerosis formation and development. Herein, we used oil red $\mathrm{O}$ staining to evaluate the lipid content of the plaques, which represents the severity of atherosclerosis. As shown in Figures $\mathbf{2 A}, \mathbf{C}$, the lipid accumulation in the atherosclerotic plaques was more severe in the stress groups compared to the controls. Clinically, after the lipid accumulation, the inflammation response and oxidative stress started to play critical roles in the development of atherosclerotic lesions. As shown in Figures 2A,D,E, chronic stress significantly increased the accumulation of macrophages (CD68: $35.5 \pm 2.1 \%$ vs. $24.7 \pm 0.8 \%, p<0.01)$ and the expression of osteopontin proteins (osteopontin: $31.1 \pm 0.8 \%$ vs. $24.2 \pm 0.7 \%, p<0.01$ ) in the atherosclerotic plaques. The results of the RT-PCR confirmed that the expressions of the oxidative stress (p67phox, gp91 ${ }^{\text {phox }}$, and $\mathrm{p} 47^{\text {phox }}$ ), inflammation (MCP-1, TLR-2, TLR-4, and CXCR-4), and proteolysis (MMP-2, MMP-9, and Cat S)-related genes were markedly elevated in the aortas of the stressed mice compared to the control aortas (Table 3 ).

\section{Chronic Stress Prompted Vascular Senescence}

Endothelial dysfunction is a common pathological phenomenon for many cardiovascular diseases, and it often occurs before atherosclerosis. In this study, the senescence-associated $\beta$-gal staining showed that the positive staining area in the stressed mice was larger than that of the controls (Figures $\mathbf{3 A}, \mathbf{B}$ ), indicating that the chronic stress resulted in endothelial cell dysfunction. 


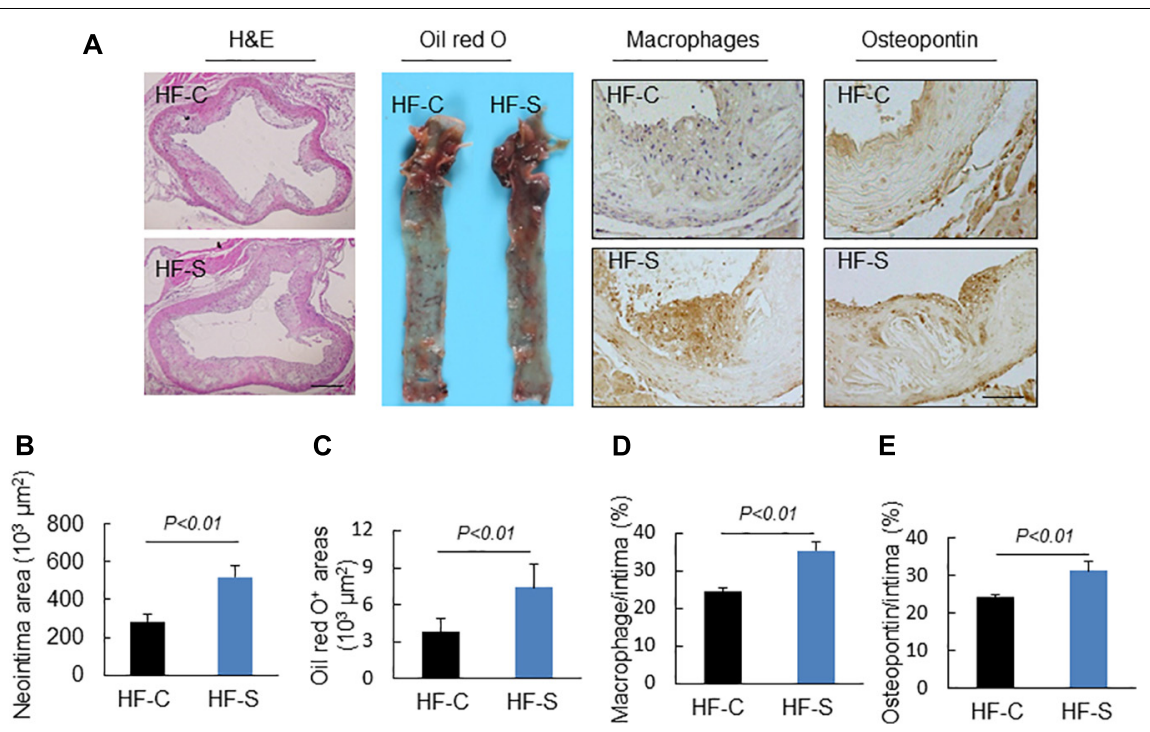

FIGURE 2 | Histological characterization of atherogenic plaques in aortic roots of two experimental groups (high-fat diet alone, HF-C; high-fat diet plus stress, HF-S). (A) Representative images applied to evaluate the neointimal hyperplasia, content of lipid-rich plaques, macrophages accumulation and osteopontin expression. (B-E) The areas for H\&E, Oil red $\mathrm{O}^{+}$and positive areas in the neointimas for osteopontin and CD68 were quantified for each section. Results are presented as neointima area, the ratio of Oil red O-stained area to the total aortic root, the ratio of the positively stained area (CD68, osteopontin) to the neointimal area in the atherosclerotic lesions. Values are presented as means \pm SEM $(n=6-7) . p<0.01$ vs. HF-C by Student's $t$-test. Scale bars: $50 \mu \mathrm{m}$.

TABLE 3 | Real-time PCR analysis of the targeted genes in the aortic roots and APN in subcutaneous fat of both experimental group mice.

\begin{tabular}{lcc}
\hline Parameter & HF-C & HF-S \\
\hline TLR-2 & $34.2 \pm 0.9$ & $41.5 \pm 1.3^{\star *}$ \\
TLR-4 & $37.8 \pm 1.9$ & $49.7 \pm 2.1^{\star *}$ \\
CXCR-4 & $71.6 \pm 3.6$ & $96.0 \pm 3.3^{\star *}$ \\
MCP-1 & $9.8 \pm 0.5$ & $15.2 \pm 0.9^{\star *}$ \\
gp 91 & $19.2 \pm 0.8$ & $26.3 \pm 0.6^{\star *}$ \\
p47 & $29.9 \pm 1.5$ & $35.8 \pm 4.4^{*}$ \\
P67 & $17.1 \pm 0.5$ & $22.7 \pm 1.0^{\star *}$ \\
Cat S & $19.6 \pm 1.5$ & $29.3 \pm 1.1^{\star *}$ \\
MMP-2 & $26.7 \pm 3.4$ & $31.0 \pm 3.5^{\star *}$ \\
MMP-9 & $22.6 \pm 3.1$ & $46.0 \pm 3.2^{\star *}$ \\
APN & $107.4 \pm 22.8$ & $69.2 \pm 19.2^{\star *}$ \\
\hline TLR-2, toll-like receptor 2; CXCR-4, C-X-C chemokine receptor-4; MMP-2, matrix \\
metalloproteinase-2; Cat S, cathepsin S; MCP-1, monocyte chemotaxis protein-1; \\
APN, adiponectin. All results are presented as means \pm SEM, ${ }^{*} P<0.05,{ }^{* * P}<0.01$ \\
vs. corresponding controls by Student's un-paired t-test.
\end{tabular}

\section{Chronic Stress Changed the Atherosclerotic Plaque Stability}

Extracellular matrix (ECM) remodeling is responsible for the stability of atherosclerotic plaques, including the synthesis and degradation of collagen, elastin, and other glycoproteins. In this study, chronic stress significantly reduced the atherosclerotic plaques' contents of collagen and $\alpha$-SMC (the main source of collagen) compared to the control group ( $\alpha$-SMC: $26.6 \pm 0.5 \%$ vs. $23.9 \pm 0.6 \%$, collagen: $31.6 \pm 1.3 \%$ vs. $19.2 \pm 1.9 \%$, respectively; $p<0.05$, $p<0.01$; Figures $3 \mathrm{~A}, \mathbf{C}, \mathbf{D})$. The results of the EVG staining demonstrated that the chronic stress significantly destroyed the integrity of elastin in the media layer compared to the control group (number of breaks: $8.2 \pm 0.3$ vs. $5.7 \pm 0.2, p<0.01$, Figures 3A,E).

\section{Pitavastatin Mitigated the Chronic Stress-Related Lipid Accumulation, Inflammatory Response, Oxidative Stress, and Plasma GLP-1/APN Axis}

At the second phase of the experiment, we evaluated the effects of a statin, i.e., pitavastatin, on stress-related atherosclerosis. Pitavastatin significantly reduced the levels of LDL-C without changing the levels of the other parameters (TG, HDL, NEFA, glucose, BUN, CREA, and body weight) (Table 4). More importantly, we observed that pitavastatin significantly improved plasma adiponectin (APN: $7904.4 \pm 664.1 \mathrm{ng} / \mathrm{ml}$ vs. $4980.3 \pm 363.7 \mathrm{ng} / \mathrm{ml}$; $p<0.01)$ and GLP-1 (GLP-1: $18.8 \pm 1.2 \mathrm{Pm}$ vs. $13.9 \pm 1.4 \mathrm{pM}$, $p<0.01)$ levels of the stressed mice as compared to control mice (Table 4).

Pitavastatin treatment also reduced atherosclerotic area compared to the stress alone group (atherosclerotic area: $496.9 \pm 28.1 \times 10^{3} \mu \mathrm{m}^{2}$ vs. $321.2 \pm 41.9 \times 10^{3} \mu \mathrm{m}^{2} ; p<0.01$; Figures 4A,B). Compared to the non-treated stress group, the group of stressed mice treated with pitavastatin exhibited significantly reduced lipid deposition, macrophage accumulation, and osteopontin expression in the stress-related atherosclerotic lesions (Figures 4A,C-E). The mRNA expressions of p67phox, p47 ${ }^{\text {phox }}$, gp91 ${ }^{\text {phox }}$, CXCR4, MCP-1, TLR-4, and TLR-2 were inhibited (Table 5). 

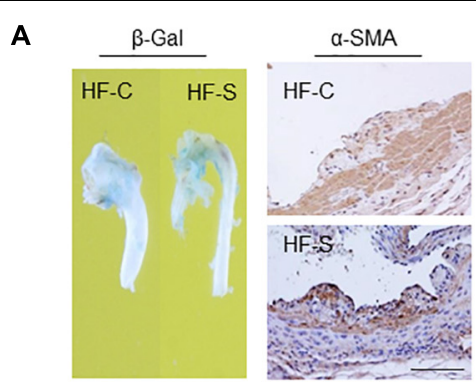

C

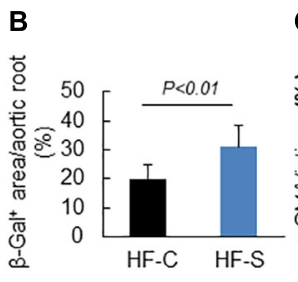

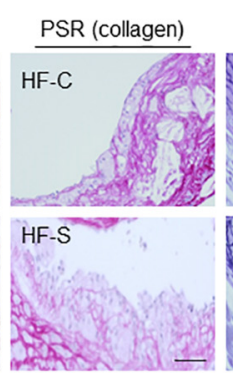

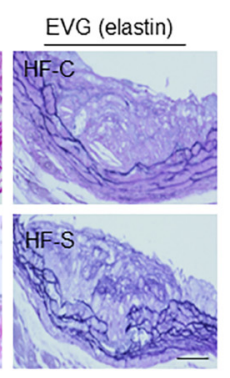

D

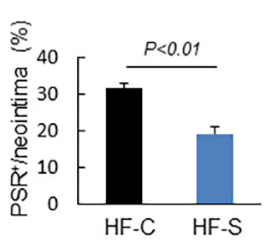

E

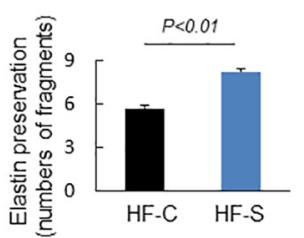

FIGURE 3 | Morphological characterization of the plaques in aortic roots of two experimental groups. (A) Representative images applied to evaluate vascular senescence, $\alpha$-SMCs, collagen, and elastin disruption. (B-E) The areas for $\beta$-Gal ${ }^{+}, \alpha-S M C s, \mathrm{PSR}^{+}$staining, and the elastic disruption degree in atherosclerotic lesions were calculated for each section. Results are presented as the ratio of $\beta$-Gal ${ }^{+}$area to the total aortic root, the ratio of the positively stained area ( $\alpha$-SMCs, $\mathrm{PSR}^{+}$) to the neointimal area in the atherosclerotic lesions, and the elastic broken numbers in the atherosclerotic lesions. Values are presented as means \pm SEM $(n=6-7) . p<0.01$ vs. HF-C by Student's $t$-test. Scale bars: $50 \mu \mathrm{m}$.

TABLE 4 | Levels of lipids activity and other biochemical parameters.

\begin{tabular}{lcc}
\hline Parameter & Stress & Pitavastatin \\
\hline TG $(\mathrm{mg} / \mathrm{dL})$ & $111.0 \pm 10.4$ & $108.5 \pm 11.2$ \\
LDL $(\mathrm{mg} / \mathrm{dL})$ & $213.5 \pm 19.6$ & $123.5 \pm 13.2^{*}$ \\
HDL $(\mathrm{mg} / \mathrm{dL})$ & $24.5 \pm 3.9$ & $26.4 \pm 3.7$ \\
NEFA ( $\mu$ EQ/L) & $773.5 \pm 20.8$ & $712.2 \pm 18.4$ \\
Glucose $(\mathrm{mg} / \mathrm{dL})$ & $159.5 \pm 13.9$ & $158.6 \pm 15.6$ \\
BUN $(\mathrm{mg} / \mathrm{dL})$ & $19.0 \pm 3.8$ & $21.5 \pm 5.1$ \\
CREA $(\mathrm{mg} / \mathrm{dL})$ & $0.5 \pm 0.0$ & $0.5 \pm 0.0$ \\
GLP-1 $(\mathrm{pM})$ & $13.9 \pm 1.4$ & $18.8 \pm 1.2^{\star *}$ \\
APN $(\mathrm{ng} / \mathrm{ml})$ & $4980.3 \pm 363.7$ & $7904.4 \pm 664.1^{\text {** }}$ \\
\hline
\end{tabular}

Abbreviations are in Table 2. APN, adiponectin. Data are presented as means \pm SEM, ${ }^{*} P<0.05,{ }^{* *} P<0.01$ vs. corresponding controls by Student's un-paired t-test.

\section{Pitavastatin Alleviated Vascular Aging and Atherosclerotic Plaque Instability}

The vascular aging was clearly alleviated by the pitavastatin treatment, as shown in Figures 5A,B. In addition, the pitavastatin treatment increased the collagen and $\alpha$-SMC contents of the atherosclerotic plaques and preserved the continuity of the elastic lamina (Figures 5A,C-E). To explore the cause of the destruction of the ECM, we determined the expressions and activities of ECM-degrading enzymes. The RT-PCR results revealed that the aortas of S-Pis mice had decreased levels of MMP-2, MMP-9, and Cat $S$ mRNAs (Table 5). In addition, the gelatinolytic activity of MMP-9 and MMP-2 was also inhibited by pitavastatin treatment (Figures 6A,B).

It is known that APN is produced mainly by adipose tissue, and we therefore investigated the mRNA expression in the subcutaneous fat of the three groups of mice. As shown in Tables 3, 5, the chronic stress significantly inhibited the APN expression of the subcutaneous fat of the mice, and the pitavastatin treatment ameliorated the expression of APN. We also observed that the pitavastatin treatment mitigated the alterations in the senescence-related proteins (p16, p21, Sirt-1, and AdipR-1) (Figures 6C,D). We used an APN neutralizing antibody to test the anti-atherosclerotic effect of pitavastatin in the stressed mice, and the results showed that this antibody abolished the protective effects of pitavastatin in stress-related atherogenesis (Table 6). In vitro, exenatide as a GPL-1 receptor agonist increased APN gene expression in cultured immature adipocytes in a dose-dependent manner (Figure 6E).

\section{DISCUSSION}

In addition to conventional cardiovascular risk factors such as high cholesterol, hypertension, and diabetes mellitus, chronic stress is now considered a risk factor for cardiovascular diseases. Explorations of the underlying mechanisms and the identification of pharmacotherapeutic targets for clinical use are thus of great significance. Our present study's findings may contribute to this field, as we observed the following: (a) Chronic stress significantly enhanced the inflammation action and oxidative stress process in $A p o E^{-/-}$mice fed a high-fat diet, and it increased endothelial senescence, which promoted the development of atherosclerosis. (b) Chronic stress favored the formation of vulnerable plaques by changing the components of the ECM, and it decreased the plaques' $\alpha$-SMC content. (c) As expected, the traditional anti-atherosclerosis drug pitavastatin alleviated the progression of atherosclerosis and promoted the 
A
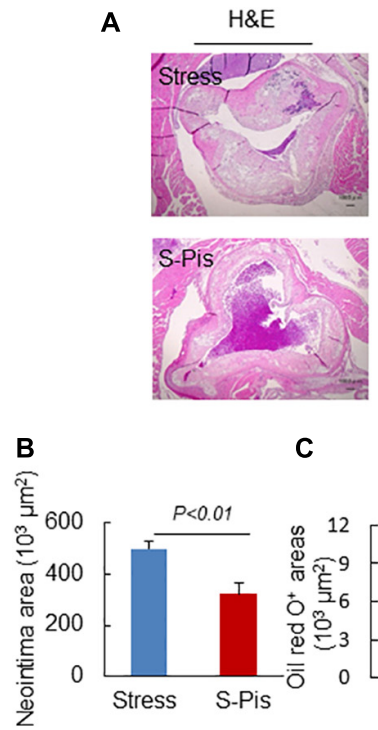

C
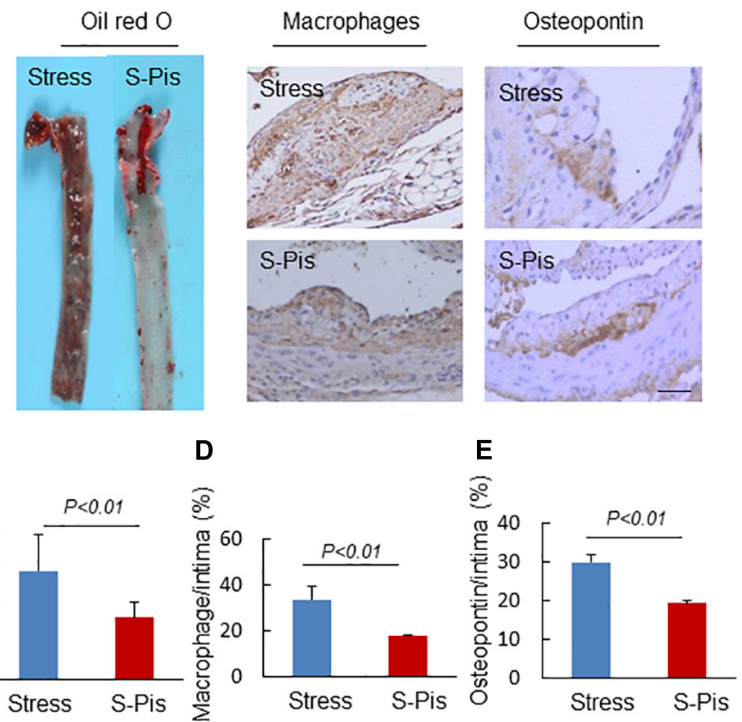

FIGURE 4 | Morphological characterization of atherosclerotic plaques in aortic roots of the stress-alone and stress plus pitavastatin (S-Pis) groups.

(A) Representative images used to assess the neointimal hyperplasia, content of lipid-rich plaques, macrophages accumulation and osteopontin expression. (B-E) The areas for $\mathrm{H} \& \mathrm{E}$, Oil red $\mathrm{O}^{+}$, and positive areas in atherosclerotic neointimas for CD68 and osteopontin were calculated for each section. Results are expressed as neointima area, the ratio of Oil red O-stained area to the total aortic root, the ratio of the positively stained area (CD68, osteopontin) to the neointimal area in the atherosclerotic lesions. Values are presented as means \pm SEM $(n=6-7)$. $p<0.01$ vs. Stress group by Student's $t$-test. Scale bars: $50 \mu \mathrm{m}$.

TABLE 5 | Real-time PCR analysis of the targeted genes in the aortic roots and APN in subcutaneous fat of both experimental group mice.

\begin{tabular}{|c|c|c|}
\hline Parameter & Stress & Pitavastatin \\
\hline TLR-2 & $40.8 \pm 2.6$ & $22.3 \pm 1.7^{\star \star}$ \\
\hline TLR-4 & $46.7 \pm 2.2$ & $19.7 \pm 0.9^{\star \star}$ \\
\hline CXCR-4 & $87.0 \pm 5.7$ & $54.2 \pm 4.0^{\star \star}$ \\
\hline gp $91^{\text {phox }}$ & $27.1 \pm 1.8$ & $16.8 \pm 0.9^{\star \star}$ \\
\hline p47phox & $32.7 \pm 2.1$ & $20.0 \pm 1.1^{\star \star}$ \\
\hline P67 phox & $23.6 \pm 1.3$ & $15.2 \pm 0.8^{\star \star}$ \\
\hline Cat S & $26.9 \pm 2.3$ & $11.3 \pm 1.1^{\star \star}$ \\
\hline MMP-2 & $27.9 \pm 3.6$ & $24.1 \pm 6.5^{\star}$ \\
\hline MMP-9 & $39.8 \pm 3.3$ & $21.4 \pm 0.8^{\star \star}$ \\
\hline APN & $76.4 \pm 11.3$ & $125.3 \pm 27.4^{\star \star}$ \\
\hline
\end{tabular}

Abbreviations are in Table 3. Data are presented as means \pm SEM, ${ }^{*} P<0.05$, ${ }^{* *} P<0.01$ vs. corresponding controls by Student's un-paired t-test.

stability of atherosclerotic plaques by ameliorating the vascular aging, inhibiting inflammation action and oxidative stress, and this effect may be exerted partly through the modulation of GLP$1 / \mathrm{APN}$ axis.

Atherosclerosis as an inflammatory disease and a great deal of evidence has confirmed the fundamental role of inflammation in the process of atherosclerosis (Zhu et al., 2018). The results of the present study demonstrated that chronic (12-week) stress significantly increased the accumulation of macrophages and the expression of osteopontin proteins of atherosclerotic plaques; the stress also increased the mRNA levels of TLR2, TLR-4, MCP-1, and CXCR-4 in the mouse aorta. The inflammatory effects on atherosclerosis of these parameters have been fully proved by previous studies (Chiba et al., 2002; Cole et al., 2010; Cheng et al., 2011). Coincidentally, it was reported that statin treatment of $A p o E^{-/-}$mice inhibited an acute stress-related inflammation of atherosclerotic plaques (Janssen et al., 2015). In agreement with several previous studies (Isingrini et al., 2011; Hayashi et al., 2014; Heidt et al., 2014), the present data indicate that chronic stress accelerated the process of atherosclerosis by enhancing inflammatory action.

The negative effects of oxidative stress in atherosclerosis have been described, including endothelial cell dysfunction, foam cell formation, plaque disruption and their interaction with inflammation, all of which contribute to atherosclerosis (Linton et al., 2000; Lankin and Tikhaze, 2017). Our present findings revealed that chronic stress significantly increased the mRNA expressions of gp91 phox p47 phox, and $067^{\text {phox }}$ in the aorta tissue. p67phox, p47 phox , and $g \mathrm{p} 91^{\text {phox }}$ are the components of $\mathrm{NAD}(\mathrm{P}) \mathrm{H}$ oxidases, which are the main source of reactive oxygen species (ROS) (Cai et al., 2003). It has been demonstrated that $\mathrm{gp} 91^{\text {phox }}$ and $\mathrm{p} 47^{\text {phox }}$ play critical roles in the development of atherosclerosis (BarryLane et al., 2001; Sorescu et al., 2002). In our present investigation, $\beta$-gal galactosidase staining revealed that chronic stress promoted the senescence of endothelial cells. Based on our present data, we speculate that chronic stress affects atherosclerosis in part by increasing the production of oxidative stress.

Plaque rupture is the leading cause of acute cardiovascular events, and such ruptures are always due to the formation of vulnerable plaques. An excessive inflammatory response, oxidative stress, and degradation of the ECM are all responsible 

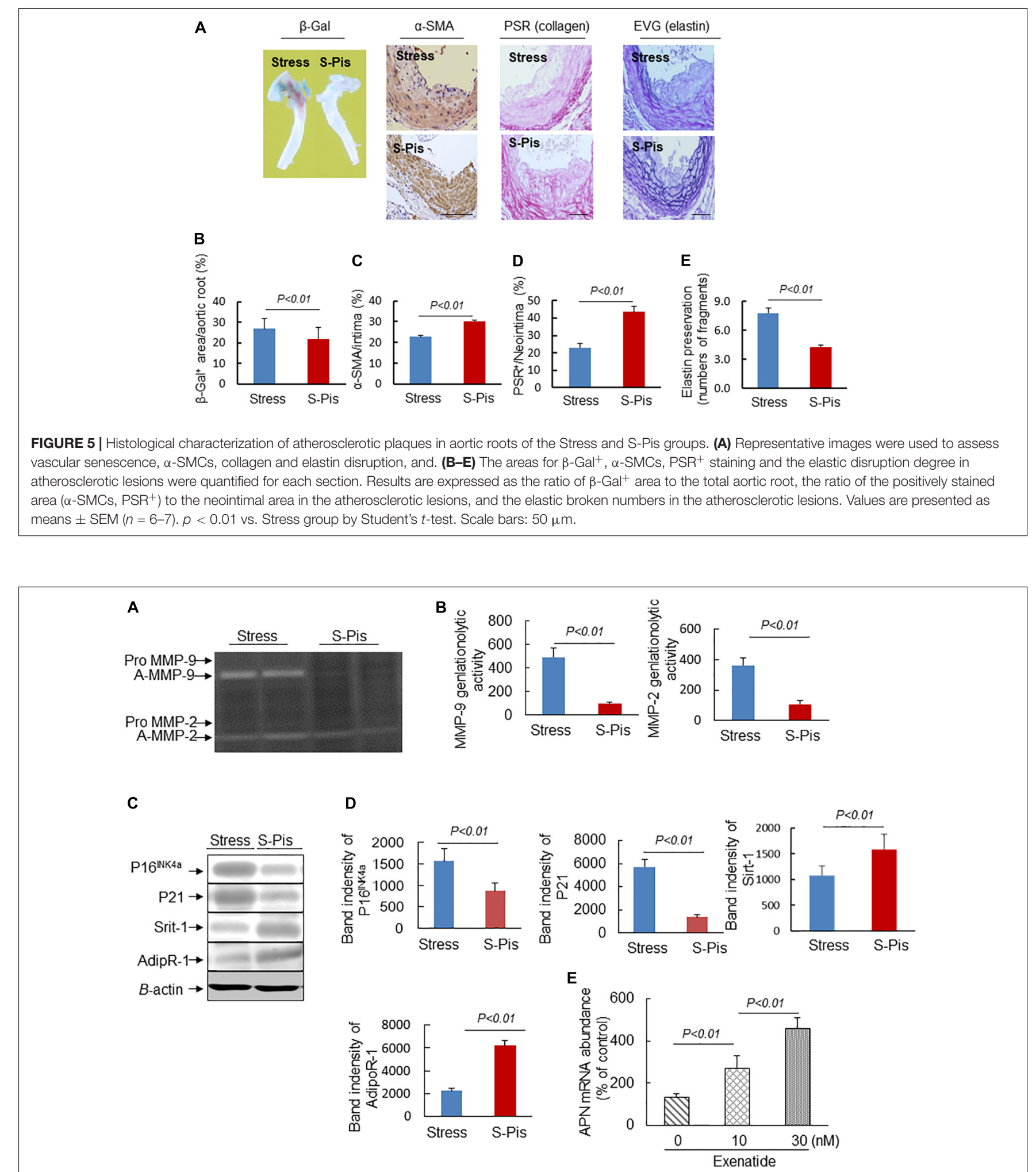

FIGURE 6 | Pitavastatin mitigated MMP-2/-9 expression/activity and targeted protein expressions in the aortic roots. (A,B) Representative gelatin zymography images and quantitative data for MMP-9 and MMP-2 activities. (C,D) Representative Western blot images and quantitative data show the protein expressions of P16 INK 4a , P21, Sirt-1, and Adip R-1. (E) Exenatide elevated APN gene expression in the stressed mouse inguinal adipose tissue-derived immature adipocytes. Values are presented as means \pm SEM $(n=3-6) . p<0.05, p<0.01$ vs. Stress group by Student's $t$-test and one-way ANOVA followed by Tukey post hoc tests. 
TABLE 6 | Histological characterization of atherosclerotic lesions in the aortic roots of both experimental group mice.

\begin{tabular}{lcc}
\hline Parameter (Aortic root, $\boldsymbol{n}=6)$ & S-Pis-C & S-Pis-NAPN \\
\hline Intima $\left(\times 10^{3} / \mu \mathrm{m}^{2}\right)$ & $308.4 \pm 19.3$ & $335.1 \pm 4.2^{*}$ \\
Media $\left(\times 10^{3} / \mu \mathrm{m}^{2}\right)$ & $201.2 \pm 26.0$ & $219.3 \pm 17.1$ \\
Intima/media ratio & $1.6 \pm 0.4$ & $1.8 \pm 0.6^{\star}$ \\
\hline
\end{tabular}

The ratio of intima to media was expressed as the ratio of intimal to media area in lesional cross-sections of heart aortic roots of HF-diet loaded stressed mice received pitavastatin $(1 \mathrm{mg} / \mathrm{kg} / \mathrm{d}$ ) plus rabbit lgG (Control, $450 \mu \mathrm{g} / \mathrm{kg} / \mathrm{d}$, S-Pis-C) or rabbit neutralizing APN antibody $(450 \mu \mathrm{g} / \mathrm{kg} / \mathrm{d}$, S-Pis-NAPN). Data are presented as means $\pm S E M$, ${ }^{*} P<0.05$ was considered statistically significant.

for the formation of vulnerable plaques (Quesada et al., 2015; Ruddy et al., 2016; Morariu et al., 2019). As mentioned before, chronic stress promoted the progression of atherosclerosis in a mouse model by enhancing inflammation and oxidative stress, and the enhanced inflammation and oxidative stress can also change the characteristics of atherosclerotic plaques and promote ruptures. We also observed that chronic stress markedly decreased the atherosclerotic plaques' collagen and $\alpha$-SMC contents. The elastin integrity of the elastic laminae was destroyed under the condition of chronic stress, and it is known that the elastin, collagen and $\alpha$-SMC contents are responsible for the stability of atherosclerotic plaques (Kumar et al., 2016; Yurdagul et al., 2016). Our results therefore indicate that the chronic stress changed the properties of the plaques, inducing the formation of vulnerable plaques.

Regarding the underlying mechanisms, we found that the chronic stress promoted the expressions of collagendegrading enzymes (i.e., MMP-2, MMP-9, and Cat S) and also increased the activity of MMP-2 and MMP9. The main function of members of the MMP (matrix metalloproteinase) family is to degrade and deposit structural proteins within the ECM, which would affect the plaque stability (Amin et al., 2016). Similarly to the MMP family, the cysteine proteases (which have collagenolytic and elastolytic activities) also involve in ECM degradation in the process of atherosclerosis-especially Cat $\mathrm{K}$ and Cat $\mathrm{S}$ (Sukhova et al., 2003; Lutgens et al., 2006). In the present study we noted that not only the expressions of MMPs and Cats but also the activities of MMP-2 and MMP-9 were significantly increased by chronic stress, suggesting that chronic stress changed the properties of atherosclerotic plaques via ECM-degrading enzymes.

The anti-atherosclerotic effects of statins have been well confirmed by basic research and in clinical practice. In addition to statins' ability to reduce LDL-C levels, it has been demonstrated that statins exert other anti-atherosclerotic effects, including anti-inflammation, improving endothelial function, and reducing the production of ROS (Jarvisalo et al., 1999; Ascer et al., 2004; Ekstrand et al., 2015). The present study is the first to investigate the anti-inflammation and anti-oxidative stress effects of statins in stress-related atherosclerosis. Pitavastatin reduced the LDL-C levels in stressed $A p o E^{-/-}$mice and we also observed that: (a) Pitavastatin decreased the inflammatory response of the atherosclerosis by reducing the accumulation of macrophages and the expression of osteopontin proteins. The mRNA levels of CXCR4, MCP-1, and TLR-2/-4 of the atherosclerotic plaques were also decreased. (b) Pitavastatin decreased the mRNA expressions of gp91 phox, $\mathrm{p} 47^{\text {phox }}$, and p67phox , all of which are related to oxidative stress. (c) Pitavastatin enhanced the stability of atherosclerotic plaques by increasing the collagen and $\alpha$-SMC contents in the plaques, and it reduced the expressions and/or activities of ECM-degrading enzymes (MMP-2, MMP-9, and Cat S), preserving the integrity of the elastic laminae. All of these effects of pitavastatin contributed to the alleviation of stress-related atherosclerosis, beyond lowering the LDL-C level. (d) Pitavastatin rectified the alterations in the senescence-related protein levels ( $p 61$, p21, and Sirt-1).

We studied the potential mechanisms underlying the effects of the chronic stress on atherosclerosis, and it is noteworthy that the pro-atherosclerosis effect of chronic stress was accompanied by a change in the level of GLP-1 and APN. Adipose tissue has been recognized as an important endocrine organ, capable of secreting various endocrine factors that modulate a wide variety of physiological functions (Fang and Judd, 2018). APN is one of the adipokines that has exhibited anti-inflammatory properties and an endothelial cell-protective effect. Several studies confirmed that APN can activate AMP kinase and $\mathrm{NF \kappa B}$ activity in human aortic endothelial cells under hyperglycemic conditions, and intervention with APN resulted in a marked reduction of the atherogenic plaque area on the abdominal aorta in a murine model (Wu et al., 2017). Together with our research, the above-described findings indicate that chronic stress induced a significant decrease in the APN level, resulting in the aggravation of atherosclerosis. We also observed that the expression of AdipR-1 in the aortic root was reversed by the pitavastatin treatment and APN neutralizing antibody abolished the protective effects of pitavastatin in stress-related atherogenesis. Moreover, in immature adipocytes, GLP-1 receptor activation by the exenatide stimulated APN expression in a dose-dependent manner. We therefore speculate that the protective effect of pitavastatin in chronic stressrelated atherosclerosis may depend in part on the GLP$1 / \mathrm{APN}$ pathway.

One major potential limitation of the present study is that although current studies contains in vivo APN depletion experiments and GLP-1-mediated regulation of APN expression in immature adipocytes, we could not conduct used genetic GLP-1 and APN animals to provide a direct evidence for the link between vascular aging and atherosclerosis and GLP-1/APN axis. Furthermore, it was proposed that chronic stress accelerated the oxidative stress of atherosclerotic lesions. Our findings for the oxidative stress were only based on the quantitative RTPCR data analysis of the components of $\mathrm{NAD}(\mathrm{P}) \mathrm{H}$ oxidases. Additional data including the actual readout for oxidative stress in the vascular lesions the rescuing lesions in the presence of antioxidants, and an alteration in mitochondrial oxidative stress levels will be more convincing for exploring 
proposed mechanisms. Further study will be needed to investigate these issues.

\section{CONCLUSION}

We observed that chronic stress aggravated high fat dietinduced atherosclerosis in a mouse model by inhibiting the GLP-1-mediated APN/adipoR1 pathway, and it subsequently enhanced the inflammation and oxidative stress process and changed the properties of atherosclerotic plaques. As anticipated, treatment with pitavastatin ameliorated this stress-related atherosclerosis. A greater understanding of the precise effects of chronic stress is of great importance for combating stress-related disorders. Although there are limitations to our present findings in mice, they demonstrate the influence of chronic stress on atherosclerosis, including potential mechanisms. Moreover, the beneficial effects of statins on chronic stress-related atherosclerosis were confirmed for the first time, which is meaningful in clinical treatment.

\section{DATA AVAILABILITY STATEMENT}

The original contributions presented in the study are included in the article/supplementary material, further inquiries can be directed to the corresponding author/s.

\section{REFERENCES}

Amin, M., Pushpakumar, S., Muradashvili, N., Kundu, S., Tyagi, S. C., Sen, U., et al. (2016). Regulation and involvement of matrix metalloproteinases in vascular diseases. Front. Biosci. 21, 89-118. doi: 10.2741/4378

Ascer, E., Bertolami, M. C., Venturinelli, M. L., Buccheri, V., Souza, J., Nicolau, J. C., et al. (2004). Atorvastatin reduces proinflammatory markers in hypercholesterolemic patients. Atherosclerosis 177, 161-166. doi: 10.1016/j. atherosclerosis.2004.07.003

Barik, J., Marti, F., Morel, C., Fernandez, S. P., Lanteri, C., Godeheu, G., et al. (2013). Chronic stress triggers social aversion via glucocorticoid receptor in dopaminoceptive neurons. Science 339, 332-335. doi: 10.1126/science.1226767

Barry-Lane, P. A., Patterson, C., van der Merwe, M., Hu, Z., Holland, S. M., Yeh, S. M. H., et al. (2001). p47phox is required for atherosclerotic lesion progression in ApoE(-/-) mice. J. Clin. Invest. 108, 1513-1522. doi: 10.1172/JCI11927

Bernberg, E., Ulleryd, M. A., Johansson, M. E., and Bergstrom, G. M. (2012). Social disruption stress increases IL-6 levels and accelerates atherosclerosis in ApoE-/mice. Atherosclerosis 221, 359-365. doi: 10.1016/j.atherosclerosis.2011.11.041

Cai, H., Griendling, K. K., and Harrison, D. G. (2003). The vascular NAD(P)H oxidases as therapeutic targets in cardiovascular diseases. Trends Pharmacol. Sci. 24, 471-478. doi: 10.1016/S0165-6147(03)00233-5

Cheng, X. W., Song, H., Sasaki, T., Hu, L., Inoue, A., Bando, Y. K., et al. (2011). Angiotensin type 1 receptor blocker reduces intimal neovascularization and plaque growth in apolipoprotein E-deficient mice. Hypertension 57, 981-989. doi: 10.1161/HYPERTENSIONAHA.110.168385

Chiba, S., Okamoto, H., Kon, S., Kimura, C., Murakami, M., Inobe, M., et al. (2002). Development of atherosclerosis in osteopontin transgenic mice. Heart Vessels 16, 111-117. doi: 10.1007/s003800200005

Cole, J. E., Georgiou, E., and Monaco, C. (2010). The expression and functions of toll-like receptors in atherosclerosis. Med. Inflamm. 2010:393946. doi: 10.1155/ 2010/393946

Cox, S. S., Speaker, K. J., Beninson, L. A., Craig, W. C., Paton, M. M., Fleshner, M., et al. (2014). Adrenergic and glucocorticoid modulation of the sterile

\section{ETHICS STATEMENT}

The animal study was reviewed and approved by the Nagoya University Graduate School of Medicine.

\section{AUTHOR CONTRIBUTIONS}

YL: main contributor to the collection and assembly of data, manuscript drafting, biological and morphological analyses, data statistical analysis, and interpretation. LP, GY, AI, and XL: collection and assembly of samples and data. QC, HW, and MK: financial support and editing of the manuscript. XWC: main contributor to financial support, design, and editing of the manuscript. All authors approved the final version of submission.

\section{FUNDING}

This work was supported in part by grants from the National Natural Science Foundation of China (Nos. 81770485, 81560240, 81800380 , and 82060052), a grant from the Young Investigation Foundation of Yanbian University (No. 2019-39) and Fudan University Hospital (No. 2018ZSQN03), "135" science and technology project of Jilin Provincial Department of Education (No. JJKH20200528KJ), and a grant from the Ministry of Education, Culture, Sports, Science, and Technology of Japan (No. 20H03574).

inflammatory response. Brain Behav. Immun. 36, 183-192. doi: 10.1016/j.bbi. 2013.11.018

Ekstrand, M., Gustafsson Trajkovska, M., Perman-Sundelin, J., Fogelstrand, P., Adiels, M., Johansson, M., et al. (2015). Imaging of intracellular and extracellular ROS levels in atherosclerotic mouse aortas ex vivo: effects of lipid lowering by Diet or Atorvastatin. PLoS One 10:e0130898. doi: 10.1371/journal. pone. 0130898

Fang, H., and Judd, R. L. (2018). Adiponectin regulation and function. Compr. Physiol. 8, 1031-1063. doi: 10.1002/cphy.c170046

Hao, C., Shintani, S., Shimizu, Y., Kondo, K., Ishii, M., Wu, H., et al. (2014). Therapeutic angiogenesis by autologous adipose-derived regenerative cells: comparison with bone marrow mononuclear cells. Am. J. Physiol. Heart Circ. Physiol. 307, H869-H879. doi: 10.1152/ajpheart.00310.2014

Hayashi, M., Takeshita, K., Uchida, Y., Yamamoto, K., Kikuchi, R., Nakayama, T., et al. (2014). Angiotensin II receptor blocker ameliorates stress-induced adipose tissue inflammation and insulin resistance. PLoS One 9:e116163. doi: 10.1371/journal.pone.0116163

Heidt, T., Sager, H. B., Courties, G., Dutta, P., Iwamoto, Y., Zaltsman, A., et al. (2014). Chronic variable stress activates hematopoietic stem cells. Nat. Med. 20, 754-758. doi: 10.1038/nm.3589

Isingrini, E., Belzung, C., d'Audiffret, A., and Camus, V. (2011). Early and lateonset effect of chronic stress on vascular function in mice: a possible model of the impact of depression on vascular disease in aging. Am. J. Geriatr. Psychiatry 19, 335-346. doi: 10.1097/JGP.0b013e318202bc42

Janssen, H., Wagner, C. S., Demmer, P., Callies, S., Solter, G., Loghmani-khouzani, H., et al. (2015). Acute perioperative- stress-induced increase of atherosclerotic plaque volume and vulnerability to rupture in apolipoprotein-E-deficient mice is amenable to statin treatment and IL-6 inhibition. Dis. Model. Mech. 8, 1071-1080. doi: 10.1242/dmm.018713

Jarvisalo, M. J., Toikka, J. O., Vasankari, T., Mikkola, J., Viikari, J. S., Hartiala, J. J., et al. (1999). HMG CoA reductase inhibitors are related to improved systemic endothelial function in coronary artery disease. Atherosclerosis 147, 237-242. doi: 10.1016/s0021-9150(99)00189-6 
Kumar, S., Chen, M., Li, Y., Wong, F. H., Thiam, C. W., Hossain, M. Z., et al. (2016). Loss of ADAMTS4 reduces high fat diet-induced atherosclerosis and enhances plaque stability in ApoE(-/-) mice. Sci. Rep. 6:31130. doi: 10.1038/srep31130

Lankin, V. Z., and Tikhaze, A. K. (2017). Role of oxidative stress in the genesis of atherosclerosis and diabetes mellitus: a personal look back on 50 years of research. Curr. Aging Sci. 10, 18-25. doi: 10.2174/1874609809666160926142640

Lei, Y., Yang, G., Hu, L., Piao, L., Inoue, A., Jiang, H., et al. (2017). Increased dipeptidyl peptidase- 4 accelerates diet-related vascular aging and atherosclerosis in ApoE-deficient mice under chronic stress. Int. J. Cardiol. 243, 413-420. doi: 10.1016/j.ijcard.2017.05.062

Lin, C. P., Huang, P. H., Lai, C. F., Chen, J. W., Lin, S. J., Chen, J.-S., et al. (2015). Simvastatin attenuates oxidative stress, NF-kappaB activation, and artery calcification in LDLR-/- Mice fed with high fat diet via down-regulation of tumor necrosis factor-alpha and TNF receptor 1. PLoS One 10:e143686. doi: 10.1371/journal.pone.0143686

Linton, M. R. F., Yancey, P. G., Davies, S. S., Jerome, W. G., Linton, E. F., et al. (2000). "The role of lipids and lipoproteins in atherosclerosis," in Endotext, eds K. R. Feingold, B. Anawalt, A. Boyce, G. Chrousos, W. W. de Herder, K. Dungan, et al. (South Dartmouth, MA: MDText.com, Inc).

Lutgens, E., Lutgens, S. P., Faber, B. C., Heeneman, S., Gijbels, M. M., de Winther, M. P. J., et al. (2006). Disruption of the cathepsin K gene reduces atherosclerosis progression and induces plaque fibrosis but accelerates macrophage foam cell formation. Circulation 113, 98-107. doi: 10.1161/circulationaha.105.561449

Morariu, M., Hodas, R., Benedek, T., Benedek, I., Opincariu, D., Mester, A., et al. (2019). Impact of inflammation- mediated response on pancoronary plaque vulnerability, myocardial viability and ventricular remodeling in the postinfarction period - the VIABILITY study: protocol for a nonrandomized prospective clinical study. Medicine 98:e15194. doi: 10.1097/MD. 0000000000015194

Quesada, I. M., Lucero, A., Amaya, C., Meijles, D. N., Cifuentes, M. E., Pagano, P. J., et al. (2015). Selective inactivation of NADPH oxidase 2 causes regression of vascularization and the size and stability of atherosclerotic plaques. Atherosclerosis 242, 469-475. doi: 10.1016/j.atherosclerosis.2015.08.011

Ruddy, J. M., Ikonomidis, J. S., and Jones, J. A. (2016). Multidimensional contribution of matrix metalloproteinases to atherosclerotic plaque vulnerability: multiple mechanisms of inhibition to promote stability. J. Vasc. Res. 53, 1-16. doi: 10.1159/000446703

Sorescu, D., Weiss, D., Lassegue, B., Clempus, R. E., Szocs, K., Sorescu, G. P., et al. (2002). Superoxide production and expression of nox family proteins in human atherosclerosis. Circulation 105, 1429-1435. doi: 10.1161/01.cir.0000012917. 74432.66

Sukhova, G. K., Zhang, Y., Pan, J. H., Wada, Y., Yamamoto, T., Naito, M., et al. (2003). Deficiency of cathepsin S reduces atherosclerosis in LDL receptordeficient mice. J. Clin. Invest. 111, 897-906. doi: 10.1172/JCI14915

Wu, W., Wang, H., Jiao, G., Yue, J., and Wang, G. (2017). Aerobic exercise suppresses atherosclerosis through adiponectin-nuclear transcription factor kappaB pathway in Apolipoprotein E-deficient mice. Am. J. Med. Sci. 353, 275-281. doi: 10.1016/j.amjms.2016.11.002

Yang, G., Lei, Y., Inoue, A., Piao, L., Hu, L., Jiang, H., et al. (2017). Exenatide mitigated diet-induced vascular aging and atherosclerotic plaque growth in ApoE-deficient mice under chronic stress. Atherosclerosis 264, 1-10. doi: 10. 1016/j.atherosclerosis.2017.07.014

Yurdagul, A. Jr., Finney, A. C., Woolard, M. D., and Orr, A. W. (2016). The arterial microenvironment: the where and why of atherosclerosis. Biochem. J. 473, 1281-1295. doi: 10.1042/BJ20150844

Zhu, Y., Xian, X., Wang, Z., Bi, Y., Chen, Q., Han, X., et al. (2018). Research progress on the relationship between atherosclerosis and inflammation. Biomolecules 8:80. doi: 10.3390/biom 8030080

Conflict of Interest: The authors declare that the research was conducted in the absence of any commercial or financial relationships that could be construed as a potential conflict of interest.

Copyright (C) 2021 Lei, Cui, Yang, Piao, Inoue, Wu, Li, Kuzuya and Cheng. This is an open-access article distributed under the terms of the Creative Commons Attribution License (CC BY). The use, distribution or reproduction in other forums is permitted, provided the original author(s) and the copyright owner(s) are credited and that the original publication in this journal is cited, in accordance with accepted academic practice. No use, distribution or reproduction is permitted which does not comply with these terms. 